\title{
13 Social cohesiveness and neighbourhood environmental deprivation: how are they related to life satisfaction in late life?
}

\footnotetext{
- Socially cohesive neighbourhood environments facilitate social inclusion; deprived neighbourhood environments do not

- Most older Europeans live in environmentally satisfactory neighbourhoods and have socially cohesive relationships with neighbours

- There is little variation between countries in regard to social cohesiveness and neighbourhood deprivation; differences on these measures seem to be driven by micro-level factors

- Residents of socially cohesive neighbourhoods report greater life satisfaction, especially those living in otherwise deprived neighbourhoods
}

\subsection{Social cohesiveness and neighbourhood deprivation}

Neighbourhood environment, particularly the extent of social cohesiveness and the physical state of the neighbourhood (e.g. cleanliness and safety), has been shown to influence well-being among older adults (Cagney et al. 2009). Social cohesiveness, as demonstrated by the presence of supportive neighbourhood-based social ties, is an indicator of social inclusion. Correspondingly, a lack of cleanliness or safety in the local area, which may be a sign of neighbourhood deprivation, is an indicator of social exclusion.

Meaningful social interactions and social reciprocity in the neighbourhood facilitate constructive aging-in-place as well as promoting positive aging experiences (Emlet \& Moceri 2012). Perceived social cohesion within neighbourhood settings and a sense of belonging are both related to enhanced well-being outcomes among older adults (Bowling et al. 2002, Momtaz et al. 2014). Social cohesiveness has also been shown to promote positive social interactions and a greater sense of inclusion (Cramm et al. 2013). Moreover, older adults who maintain relationships with their neighbours have elevated feelings of belonging and self-worth, as well as greater overall life satisfaction (Oswald et al. 2011).

The physical environment of a neighbourhood may either enhance or restrain the well-being of older adults. Safe and well-organized neighbourhoods promote 
social engagement outside of the home and, as such, are reflective of social inclusion. Research shows that living in nice and safe neighbourhoods improves well-being in later life (Netuveli et al. 2006). In contrast, living in environmentally deprived neighbourhoods is negatively related to well-being among older adults (Scharf et al. 2002). Neighbourhoods with high rates of vandalism, graffiti and unkempt streets reflect social exclusion insofar as these particular characteristics tend to limit the actions and the behaviours of people living in such settings (Scharf et al. 2002).

A key question that is yet to be resolved is whether feelings of social cohesiveness among neighbours counteract the otherwise negative effects of residing in deprived neighbourhoods. Research has underscored a positive link between neighbourhood social connections and satisfaction (Hur \& Morrow-Jones 2008). Some findings suggest that neighbourhood social cohesion has a mediating role in the generally negative association between neighbourhood deprivation and individual mental health outcomes (Drukker \& van Os 2003). Additional empirical work is required, therefore, in order to ascertain whether the association between neighbourhood social cohesion and well-being varies according to the degree of neighbourhood deprivation. That is, do social ties become even stronger in their association with well-being when older adults live in environmentally deprived neighbourhoods and, in essence, assist in overcoming the countervailing effects of social exclusion that stem from the physical environment?

The study reported upon in this chapter explores the nature and extent of social cohesion and neighbourhood deprivations among older Europeans. In the first stage, a component representative of social cohesion and a component representative of neighbourhood deprivation were developed using data from the social exclusion module in SHARE Wave 5. Social cohesion was operationalised as a measure of the strength of relational ties with neighbours. Neighbourhood deprivation was operationalised as a measure of environmental characteristics representative of the physical quality of the neighbourhood. In the second stage, country differences of each component were analysed to explore whether or not variations on these domains exist between countries. The third and final phase of the analysis examined how social cohesion and neighbourhood deprivation respectively influence subjective well-being, operationalised as overall life satisfaction. In addition, the analysis builds on these findings to consider if and how the interaction of social cohesion and neighbourhood deprivation alters the associations with life satisfaction. 


\subsection{Social inclusion and social exclusion}

The study addressed the neighbourhood aspects of the social exclusion module from SHARE Wave 5. Household survey respondents eligible for the social exclusion module $(n=41,784)$ were asked to rate the level to which they feel part of the local area (hh022_), if vandalism and graffiti are a big problem in the neighbourhood (hh023_), the extent of neighbourhood cleanliness (hh024_) and if local people would be helpful if they would be in trouble (hh025_). The variables are coded on a 4-point Likert scale ranging from 1) strongly agree to 4) strongly disagree. The probe on vandalism and graffiti was reverse coded to align the positive answer category order with the other variables in the question series. For the purpose of the present analysis, the four variables were separated into two components each consisting of two items: social cohesion (hh022_; hh025_) which reflects social inclusion, and neighbourhood deprivation (hh023_; hh024_), which represents social exclusion. Dummy variables were created to distinguish between those answering strongly agree or agree (1) and those answering strongly disagree or disagree (0). For the social cohesion component, a count of the strongly agree or agree category (1) was calculated. High values on the possible score of $0-2$ represent more social inclusion. For the neighbourhood deprivation component, a count of the strongly disagree or disagree category (0) was calculated. High values on the possible score of $0-2$ represent greater exclusion.

Descriptive analysis of the sample showed that the surveyed older Europeans lived predominantly in neighbourhoods in which they felt high levels of social cohesiveness. The majority had a score of two in this variable (81.5\%), suggesting they felt a part of their local area and would have people willing to help them if they encountered any troubles. 15 per cent (15.2\%) had this level of agreement with only one of the two social cohesion items. Between three and four per cent (3.3\%) did not agree with either statement, an indicator of having no feelings of social cohesiveness with neighbours.

Similarly, the sample of older Europeans lived in neighbourhoods without much perceived deprivation. That is, members of the sample resided predominantly in neighbourhoods which were seen to be clean, safe and subject to little vandalism. 73 per cent had a score of zero on the neighbourhood deprivation variable. 21 per cent had a score of one, suggestive of neighbourhood environments with some degree of deprivation. Some six per cent of respondents had the highest score of two, indicating that they lived in local areas with high levels of neighbourhood environment deprivation. 


\subsection{Social cohesion and neighbourhood deprivation: country comparisons}

Analysis of country differences revealed significant variations on neighbourhood social cohesion and neighbourhood deprivation (see Figures 13.1 and 13.2). For neighbourhood social cohesion, a one-way analysis of variance (ANOVA) yielded significant results with a small to medium effect size, $F(14,40,629)=50.87, p<0.001$, Cohen's $\mathrm{f}^{2}=0.13$. On the whole, respondents from Israel reported the lowest country mean (1.68), those from Sweden reported the highest mean (1.89), and the remaining 13 countries fell in between these two extremes. However, as is demonstrated in Figure 13.1, post hoc analysis using the Tukey HSD criterion revealed no unique or distinct country subgroupings.

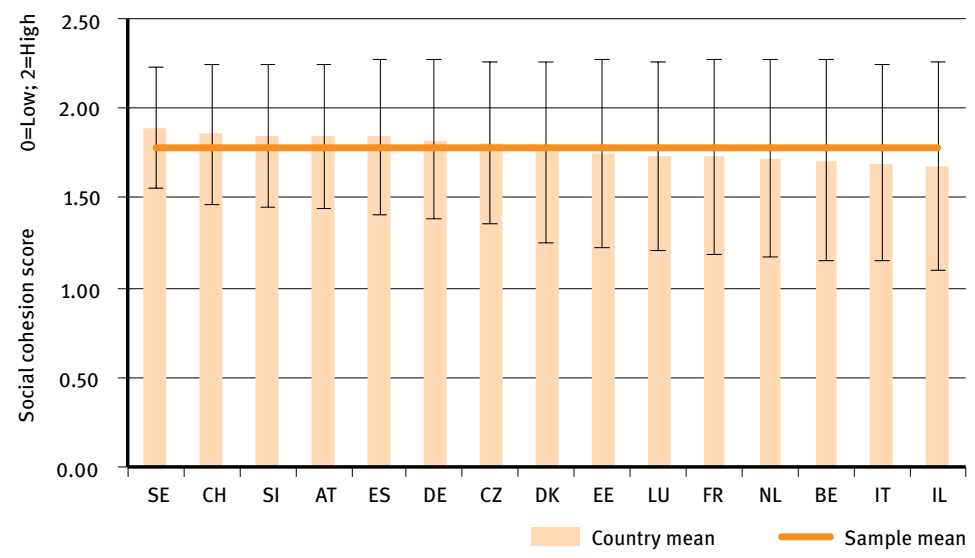

Figure 13.1: The extent of neighbourhood social cohesion, by country

Notes: Countries ordered according to mean, $\mathrm{n}=40,629$, unweighted, $\mathrm{F}(14,40,628)=50.87$, $\mathrm{p}=.000$, Cohen's $\mathrm{f} 2=.13$

Source: SHARE Wave 5 release 0

For neighbourhood deprivation, ANOVA results of country differences were significant with a medium effect size, $F(14,40,730)=205.20, p<0.001$, Cohen's $\mathrm{f}^{2}=0.26$. In addition, the Tukey HSD post hoc analysis revealed that distinct country variation for perceived neighbourhood deprivation could be discerned. As Figure 13.2 shows, three distinct country sub-groupings with relatively high levels of deprivation emerged. Respondents from the Czech Republic reported the highest mean of neighbourhood deprivation (0.72). The corresponding mean of the Italian respondents was 0.54 , forming the second country grouping. Israeli respondents had a mean of 0.46 and formed a third distinct country grouping. A 
distinct country sub-grouping also surfaced for the lowest neighbourhood deprivation score. The Swedish respondents (0.12) reported having the lowest neighbourhood deprivation score of all the respondents in this 15-country comparison.

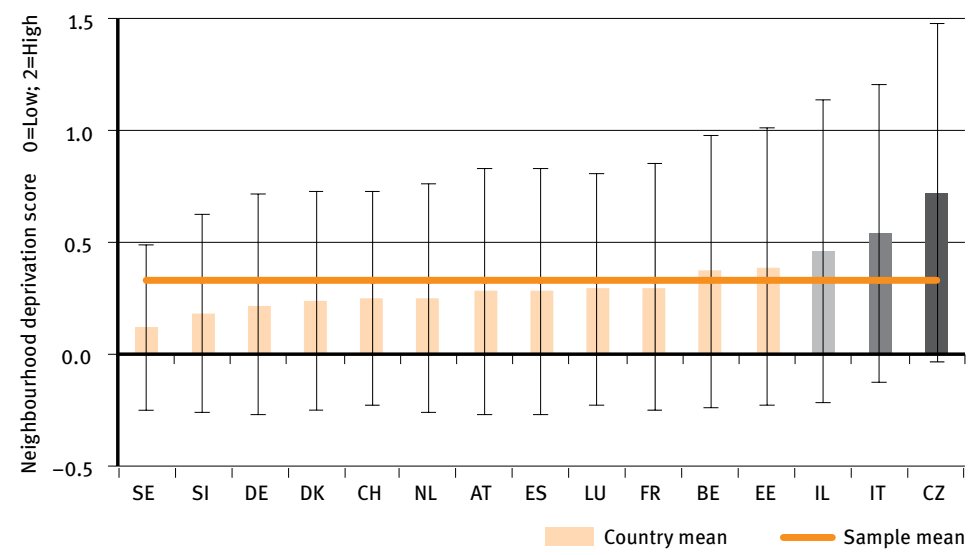

Figure 13.2: The extent of neighbourhood deprivation, by country

Notes: Countries ordered according to mean, $\mathrm{n}=40,731$, unweighted, $\mathrm{F}(14,40,730)=205.20$, $\mathrm{p}=.000$, Cohen's $\mathrm{f} 2=.26$

Source: SHARE Wave 5 release 0

In sum, the general lack of distinct country differences on perceived neighbourhood social cohesiveness suggests that to the extent that the individual scores on this variable vary, it is due to individual factors rather than country-level norms and characteristics. This same conclusion can be drawn from the relatively small number of distinct country sub-groupings that emerged in relation to the neighbourhood deprivation measure as well. That is, neighbourhood exclusion may primarily reflect differences in micro-level characteristics, such as poverty status or urban/rural neighbourhood settings, rather than clear cultural, country specific variations.

\subsection{Social cohesiveness and neighbourhood deprivation: well-being}

The final analysis considered the relationship between the measures of neighbourhood social cohesion and neighbourhood deprivation and subjective well-being. It was performed as a household level analysis among the respondents who were 
eligible for the social exclusion module. Specifically, the analysis was designed to examine how social cohesiveness and neighbourhood deprivation intersect in their associations with well-being in later life. The well-being outcome was operationalised using a subjective measure of self-rated life satisfaction. In SHARE Wave 5 , respondents were asked to rate their satisfaction with their life on a scale of $0-10$, with 0 meaning completely dissatisfied and 10 meaning completely satisfied (ac012_).

The statistical analysis utilised hierarchical OLS regressions. The first step in the analysis considered the associations of neighbourhood social cohesion and neighbourhood deprivation with life satisfaction, controlling for sociodemographic background, perceived economic adequacy, urban/rural neighbourhood, country, health and personal social network characteristics. The personal social network variables included a dummy variable indicating if a respondent has no children (0) or one or more children (1); the frequency of contact with the most contacted child ranging from never (1) to daily (7); and marital status, which was operationalised as a series of dummy variables that distinguished between married/partnered, single, divorced or widowed respondents. In the second step of the analysis, an interaction term of neighbourhood social cohesion and neighbourhood deprivation was entered.

Table 13.1: The inter-relationship of neighbourhood social cohesion and neighbourhood deprivation vis-a-vis life satisfaction: hierarchical regressions

\begin{tabular}{|c|c|c|c|c|c|c|}
\hline Variables & Model 1 & & & Model 2 & & \\
\hline & Coefficient & $\begin{array}{l}\text { Standard } \\
\text { error }\end{array}$ & $\begin{array}{l}\text { Standar- } \\
\text { dised } \beta\end{array}$ & Coefficient & $\begin{array}{l}\text { Standard } \\
\text { error }\end{array}$ & $\begin{array}{l}\text { Standar- } \\
\text { dised } \beta\end{array}$ \\
\hline Social cohesion & $0.386^{\star \star \star}$ & 0.018 & 0.099 & $0.06^{\star \star \star}$ & 0.022 & 0.092 \\
\hline $\begin{array}{l}\text { Neighbourhood } \\
\text { deprivation }\end{array}$ & $-0.108^{\star \star \star}$ & 0.015 & -0.034 & $-0.193^{\star \star \star}$ & 0.043 & -0.060 \\
\hline $\begin{array}{l}\text { Social cohesion } \\
\mathrm{x} \text { neighbourhood } \\
\text { deprivation }\end{array}$ & & & & $0.051^{*}$ & 0.025 & 0.027 \\
\hline
\end{tabular}

Significance: ${ }^{\star \star \star}=1 \% ;{ }^{\star \star}=5 \% ;{ }^{*}=10 \%$ Notes: $n=36,973$, controlled for age, gender, marital status, education, perceived income adequacy, children (dummy), most frequent contact with a child, country, urban/rural setting, number of mobility limitations, physical health symptoms

Source: SHARE Wave 5 release 0 
The first model explained a respectable amount of variance in the life satisfaction outcome $(\mathrm{R} 2=0.29)$. It revealed that life satisfaction was positively associated with social cohesiveness $(\beta=0.10)$, even after controlling for background, personal social network and health characteristics. In contrast, a negative association was found between neighbourhood deprivation and life satisfaction ( $\beta=$ -0.03). Moreover, the standardised coefficients indicate that living in a socially cohesive neighbourhood had a stronger positive association with life satisfaction than the negative association of the deprived environmental characteristics did. In other words, the quality of the relationships with nearby neighbours seems to have a stronger positive impact on well-being in later life than the negative consequences of living in deprived neighbourhoods.

These findings highlight the next question addressed in the current inquiry, namely, whether the positive strength of strong social relationships with neighbours on well-being offsets the negative experience of living in physical environments which are otherwise deprived. In order to address this issue, an interaction term was entered in the second step of the analysis. The interaction term allows exploration of whether and how the two variables - social cohesiveness and neighbourhood deprivation - inter-relate in their association with late life satisfaction. The results revealed that the interaction between social cohesiveness and neighbourhood deprivation was, indeed, significantly associated with overall life satisfaction. That is, the interaction showed that the positive association between neighbourhood social cohesion and life satisfaction $(\beta=0.10)$ became stronger as neighbourhood deprivation increased, with the standardised coefficient of the interaction term increasing by 0.03 . This means that having social connections with others in the neighbourhood was even more related to life satisfaction among those older Europeans who resided in deprived local areas, that is, neighbourhoods with higher rates of crime, graffiti and unkempt streets and parks, than it was among those who lived in nicer residential areas.

\subsection{Lessons from this study}

Social inclusion and exclusion were examined in parallel in the analyses presented in this chapter utilising measures generated from the neighbourhood aspects of the social exclusion module in SHARE Wave 5. A measure of social cohesiveness, indicative of the nature of the personal relationships with neighbours, was developed to represent social inclusion. In contrast, a measure of neighbourhood deprivation was generated as a measure of social exclusion. This measure represented exposure to negative physical neighbourhood characteristics, such as vandalism. 
The descriptive analysis of these two measures revealed that the majority of survey participants lived in neighbourhoods in which there was a high level of social inclusion and limited exclusion. Personal social connection and strong interrelationships between neighbours were relatively common. Four-fifths of the older Europeans sampled indicated that they felt a part of their local area and that their neighbours would support and assist them if they would need help. However, a small but notable number of SHARE survey participants lacked one or both of the indicators of neighbourhood social cohesiveness and therefore experienced some level of social alienation within their neighbourhood setting. Likewise, a large majority of the sample lived in socially inclusive neighbourhoods in terms of their physical environmental characteristics. Such neighbourhoods were clean and safe and had little or no reported vandalism. Some degree of neighbourhood deprivation was experienced, however, by nearly one-third of the older Europeans sampled in the survey. For these persons, the neighbourhood surroundings may yield a greater sense of insecurity about physical safety and also a perception of barriers which limit their active engagement with their surroundings.

The country specific analyses of social cohesiveness and neighbourhood deprivation revealed that country differences in these measures do exist. Swedish respondents had the highest score for social cohesiveness combined with the lowest score for neighbourhood deprivation. Those from the Czech Republic had the highest score for neighbourhood deprivation but an average degree of social cohesion. Respondents from Israel emerged as having poor social inclusion on both domains; low social cohesion and high neighbourhood deprivation scores. However, the lack of distinctive country sub-groupings in the post-hoc analyses suggests that the reasons for differences in social cohesion and neighbourhood deprivation are most likely multifaceted and reflect more than country differences alone.

The multivariate findings, which examined the associations between social cohesion, neighbourhood deprivation and subjective well-being, underscore the intrinsic value of social connections in later life, particularly among older Europeans living in deprived neighbourhoods. Life satisfaction was greater for people who had stronger social ties and feelings of connectedness with their neighbours, all things considered. It was lower, on the other hand, among those living in environmentally deprived, unkept neighbourhoods in which vandalism occurred, again, after controlling for other factors. Moreover, the findings revealed that social cohesiveness and a sense of connectedness with neighbours had a more positive association with life satisfaction among older adults living in deprived neighbourhoods than among those living in less deprived neighbourhoods. In other words, strong social ties become even more important in improving sub- 
jective well-being when persons reside in neighbourhoods which may lead to feelings of being excluded from their local surroundings because of its physical environmental characteristics. In conclusion, it can be asserted that social inclusion, in terms of social cohesiveness, can play an important role in improving quality of life among older Europeans, especially within neighbourhoods that are otherwise environmentally deprived.

\section{References}

Bowling, Ann, Banister, David, Sutton, Stephan, Evans, Olga, Windsor, John (2002): "A multidimensional model of the quality of life in older age”. In: Aging \& Mental Health 6. No. 4, p. 355-371.

Cagney, Kathleen, Glass, Thomas, Skarupski, Kimberly, Barnes, Lisa, Schwartz, Brian, Mendes de Leon, Carlos (2009): “Neighborhood-level cohesion and disorder: measurement and validation in two older adult urban populations". In: Journal of Gerontology: Social Sciences 64B. No. 3, p. 415-424.

Cramm, Jane, van Dijk, Hanna, Nieboer, Anna (2013): “The importance of neighborhood social cohesion and social capital for the well being of older adults in the community". In: The Gerontologist 53. No. 1, p. 142-152.

Emlet, Charles, Moceri, Joane (2012): “The importance of social connectedness in building age-friendly communities”. In: Journal of Aging Research 2012, p. 1-9.

Drukker, Marjan, van Os, Jim (2003): “Mediators of neighbourhood socioeconomic deprivation and quality of life". In: Social Psychiatry and Psychiatric Epidemiology 38, p. 698-706.

Hur, Misun, Morrow-Jones, Hazel (2008): “Factors that influence residents' satisfaction with neighborhoods”. In: Environment and Behavior 40. No. 5, p. 619-635.

Momtaz, Yadollah, Haron, Sharifah, Ibrahim, Rahimah, Hamid, Tengku (2014): “Social embeddedness as a mechanism for linking social cohesion to well-being among older adults: moderating effect of gender". In: Clinical Interventions in Aging 4. No. 9, p. 863-870.

Netuveli, Gopalakrishnan, Wiggins, Richard, Hildon, Zoe, Montgomery, Scott, Blane, David (2006): "Quality of life at older ages: evidence from the English longitudional study of aging (wave 1)”. In: Journal of Epidemiology \& Community Health 60, p. 357-363.

Oswald, Frank, Jopp, Daniela, Rott, Christoph, Wahl, Hans-Werner (2011): “Is aging in place a resource for or risk to life satisfaction?”. In: The Gerontologist 51. No. 2, p. 238-250.

Scharf, Tom, Phillipson, Chris, Smith, Allison, Kingston, Paul (2002): Growing older in socially deprived areas: social exclusion in later life. London: Help the Aged. 
